\title{
Efectos de una dieta con alto contenido de grasas sobre patrones conductuales alimentarios
}

\author{
Daniel Díaz-Urbina, Rodrigo Erick Escartín-Pérez, Verónica Elsa López-Alonso* y Juan Manuel Mancilla-Díaz \\ Laboratorio de Neurobiología de la Alimentación, Universidad Nacional Autónoma de México, FES-Iztacala, Tlalnepantla, México \\ Recibido, marzo 27/2017 \\ Concepto de evaluación, mayo 3/2017 \\ Aceptado, junio 24/2017

\begin{abstract}
Referencia: Díaz-Urbina, D., Escartín-Pérez, R.E., LópezAlonso, V.E. \& Mancilla-Díaz, J.M. (2018). Efectos de una dieta con alto contenido de grasas sobre patrones conductuales alimentarios. Acta Colombiana de Psicología, 21(1), 95-105. doi: http://www.dx.doi.org/10.14718/ ACP.2018.21.1.5
\end{abstract}

Resumen

\begin{abstract}
El consumo excesivo de alimentos con alto contenido de grasas se ha asociado con el incremento de la obesidad. Los efectos fisiológicos y metabólicos del consumo de dietas altas en grasa han sido estudiados extensamente; sin embargo, los mecanismos conductuales asociados al desarrollo de la obesidad por el consumo de estas dietas se han explorado en menor medida. Por tanto, el objetivo del presente estudio fue caracterizar los cambios en los patrones conductuales de la alimentación producidos por el consumo de una dieta alta en grasas durante diez días. Se utilizaron ratas macho Wistar con acceso libre al alimento, asignadas a uno de dos grupos, y durante diez días estuvieron bajo una dieta alta en grasa (45\% de calorías provenientes de grasas) o una dieta estándar de laboratorio. En los días 1, 5 y 10 se realizó un análisis detallado de la conducta alimentaria al inicio del periodo de oscuridad. Los resultados mostraron que los sujetos expuestos a la dieta alta en grasa acumularon más grasa corporal y tuvieron mayor eficiencia de la alimentación que el otro grupo, sin incremento del peso corporal ni alteraciones del patrón típico de la secuencia de saciedad conductual. Esto sugiere que la exposición a dietas con alto contenido de grasas puede producir cambios conductuales antes de que se presente una ganancia de peso excesivo, lo que afecta principalmente los mecanismos de control de eficiencia alimentaria.

Palabras clave: Conducta alimentaria, saciedad, peso corporal, tejido adiposo, dieta.
\end{abstract}

\section{Effects of a high-fat diet on behavioral eating patterns}

\begin{abstract}
Excessive consumption of high-fat food has been associated with increased prevalence of obesity. The physiological and metabolic effects of high-fat diets have been extensively studied. Nevertheless, the behavioral mechanisms associated with the development of obesity induced by consumption of these diets has been less explored. Therefore, the aim of the present study was to characterize the changes in the behavioral feeding patterns produced by the consumption of a high-fat diet during 10 days. Male Wistar rats with free access to food were assigned to one of two groups, and for 10 days, they had access to a highfat diet ( $45 \%$ calories from fat) or to a standard diet. Detailed analysis of feeding behavior was performed on days 1, 5 and 10 at the beginning of the dark period. The results showed that subjects exposed to the high-fat diet accumulated more body fat and showed increased feeding efficiency, in absence of excessive body weight increase or alterations in the behavioral satiety sequence pattern. These findings suggest that exposure to high-fat diets may produce behavioral changes before excessive gain of body weight occurs, primarily affecting control mechanisms of feeding efficiency.

Key words: Feeding behavior, satiety, body weight, adipose tissue, diet.
\end{abstract}

\footnotetext{
* Av. de los Barrios 1, Los Reyes Iztacala, Tlalnepantla, Edo. de México, C. P. 54090, México. + 525556231333, ext. 39717. velopez@unam.mx La presente investigación recibió apoyo financiero de la UNAM a través de los proyectos IN 224214 (DGAPA) y FESI-DIP-PAPCA-2014-54. Agradecemos al Consejo Nacional de Ciencia y Tecnología (CONACyT) por la concesión de la beca 323985 otorgada a Daniel Díaz Urbina (Becario: 262528) para la realización de sus estudios de Doctorado.
} 


\title{
Efeitos de uma dieta com alto conteúdo de gordura sobre padróes comportamentais alimentares
}

Resumo

\begin{abstract}
O consumo excessivo de alimentos com alto conteúdo de gordura tem sido associado com o aumento da obesidade. Os efeitos fisiológicos e metabólicos do consumo de dietas altas em gordura têm sido estudados extensamente, contudo os mecanismos comportamentais relacionados com o desenvolvimento da obesidade pelo consumo dessas dietas têm-se explorado em menor medida. Portanto, o objetivo deste estudo foi caracterizar as mudanças nos padrões comportamentais da alimentação produzidas pelo consumo de uma dieta alta em gordura durante dez dias. Utilizaram-se ratos de laboratório macho Wistar, com acesso livre ao alimento, designados a um de dois grupos, e durante dez dias estiveram sob uma dieta alta em gordura (45\% de calorias provenientes de gorduras) ou uma dieta padrão de laboratório. Nos dias 1, 5 e 10, realizou-se uma análise detalhada do comportamento alimentar ao início do período de escuridão. Os resultados mostraram que os sujeitos expostos à dieta alta em gordura acumularam mais gordura corporal e tiveram maior eficiência da alimentação do que o outro grupo, sem aumento do peso corporal nem alterações do padrão da sequência de saciedade comportamental. Isso sugere que a exposição a dietas com alto conteúdo de gordura pode produzir mudanças comportamentais antes de apresentar um ganho de peso excessivo, o que afeta principalmente os mecanismos de controle de eficiência alimentar.

Palavras-chave: Comportamento alimentar, dieta, peso corporal, saciedade, tecido adiposo.
\end{abstract}

\section{INTRODUCCIÓN}

La grasa es un macronutriente importante en la dieta occidental, y en la denominada "dieta americana", es responsable del $35 \%$ de la ingesta calórica total (Hennink \& Maljaars, 2013). Una dieta rica en grasas puede conducir fácilmente a un consumo excesivo de alimento, lo que eventualmente puede llevar al desarrollo de la obesidad debido a la afectación del sistema homeostático.

Investigaciones previas con animales de laboratorio han mostrado que el consumo de dietas altas en grasa afecta componentes que forman parte del sistema homeostático, como el ritmo circadiano (Honma, Hikosaka, Mochizuki \& Goda, 2016; Sherman et al., 2012) y la actividad espontánea de los animales (Ludmilla et al., 2017). Por ejemplo, sujetos experimentales expuestos a una dieta alta en grasa presentaron un incremento inicial de la acumulación de tejido adiposo, sin que el peso corporal aumentara significativamente; sin embargo, después de tres semanas se encontró un aumento del peso corporal e hipertrofia de los adipocitos (La Fleur, Van Rozen, Luijendijk, Groeneweg \& Adan, 2010; Woods, Seeley, Rushing, D'Alessio \& Tso, 2003). Adicionalmente, se ha demostrado, con el modelo de obesidad inducida por dieta(OID), que el consumo crónico de dietas obesogénicas, además de favorecer el aumento del tejido adiposo y el incremento del peso corporal, induce alteraciones metabólicas (Sáinz, Barrenetxe, Moreno-Aliaga \& Martínez, 2015).

Dichas investigaciones sugieren que el aumento del peso corporal y del tejido adiposo se deben al sobreconsumo de dietas altas en grasa, aunque también podrían estar contribuyendo fuertemente algunos patrones conductuales asociados al consumo de alimento. Con respecto a esto, Melhorn et al. (2010) encontraron que los sujetos en una dieta alta en grasa presentaban episodios alimentarios menos frecuentes, pero de mayor duración, y que el aumento del tejido adiposo correlacionaba significativamente con el tamaño del episodio alimentario. Estos resultados aluden a la alteración de algún sistema de neurotransmisores que participa en la homeostasis energética, ya que estos parámetros conductuales son regulados a nivel cerebral.

Igualmente, estudios recientes corroboran que la ingestión crónica de dietas altas en grasa produce cambios en algunos sistemas de neurotransmisores que participan en la regulación central de los componentes motivacionales y metabólicos de la alimentación, como lo son el sistema dopaminérgico (Carlin et al., 2016; Fordahl, Locke \& Jones, 2016), serotoninérgico (Yu et al., 2013) y el de las melanocortinas (Chandler, Viana, Oswald, Wauford \& Boggiano, 2005; La Fleur et al., 2010). En consecuencia, estos sistemas de neurotransmisión han sido asociados con aspectos específicos de la regulación de la conducta alimentaria (Leibowitz \& Alexander, 1998; Mul, Spruijt, Brakkee \& Adan, 2013; Terry, Gilbert \& Cooper, 1995).

Desde el punto de vista fisiológico, los mecanismos que controlan la ingesta de alimento y la saciedad son complejos, pues el desarrollo de la saciedad (inhibición de la ingesta de alimento) inicia en el tracto gastrointestinal a través de dos vías: en la primera, la distensión mecánica del estómago envía señales a través del núcleo del tracto solitario hacia los centros de saciedad en el sistema nervioso central; $y$ en la segunda, el ingreso del alimento al intestino delgado estimula la secreción de péptidos que viajan por el 
torrente sanguíneo o activan aferentes vagales, funcionando como señales saciatorias en regiones cerebrales, como el hipotálamo (Hennink \& Maljaars, 2013).

En la condición de OID, la respuesta vagal a la distensión gástrica se reduce y los efectos de la grelina, un conocido péptido con acción orexigénica, aumentan; de forma semejante, la actividad vagal en respuesta a la entrada de nutrientes al intestino también disminuye, y la alteración de la secreción de péptidos reduce las señales de saciedad y favorece el aumento del consumo de alimento y del peso corporal (Kentish et al., 2012; Maljaars, 2013).

Por otra parte, un gran número de investigaciones se enfocan en conocer solo la cantidad de alimento ingerido (g) o el consumo de energía (kcal), sin embargo, desde la perspectiva conductual, el investigador puede realizar la medición de diferentes parámetros conductuales -como la latencia, la tasa local de alimentación, la frecuencia y la duración de los episodios, entre otros-para describir el patrón de la conducta alimentaria y determinar las causas del aumento o reducción de la ingesta de alimento debidas a la manipulación de una variable.

Los patrones de conducta alimentaria descritos se han caracterizado con el uso de técnicas como el análisis de la microestructura alimentaria y la secuencia de saciedad conductual (SSC) (López-Alonso, Mancilla-Díaz, RitoDomingo, González-Hernández \& Escartín-Pérez, 2007; Mancilla-Díaz, Escartín-Pérez, López-Alonso, FloranGarduño \& Romano-Camacho, 2005; Tallett, Blundell \& Rodgers, 2009; Tejas-Juárez et al., 2014), técnicas que dan cuenta de aspectos conductuales específicos -como el proceso de satisfacción o terminación del intervalo de alimentación y el desarrollo de la saciedad o inhibición postingestión de alimento- o inespecíficos -como la terminación del intervalo de alimentación por náuseas, dolor, sedación o hiperactividad- de la conducta alimentaria (Halford, Wanninayake \& Blundell, 1998).

Con el uso de dichas técnicas se ha demostrado, en general, que de manera natural la ingestión de alimento induce satisfacción, la cual se expresa por la presencia de una secuencia conductual altamente estereotipada, donde la alimentación es seguida de una actividad -conducta motora-, luego por el acicalamiento, y termina con el descanso (Halford et al., 1998).

Estos antecedentes muestran que el conocimiento sobre los efectos fisiológicos y metabólicos del consumo de dietas altas en grasa es extenso; sin embargo, los mecanismos conductuales asociados al desarrollo de la obesidad y el sobrepeso inducido por el consumo de estas dietas han sido menos explorados. Adicionalmente, el estudio de los efectos de las dietas con alto contenido de grasas sobre los patrones de la conducta alimentaria podría dar cuenta de los procesos motivacionales -como el hambre, el apetito, la satisfacción y la saciedad-relacionados con el desarrollo de la ganancia de peso anormal. Teniendo esto en cuenta, el objetivo de la presente investigación fue caracterizar los cambios en los patrones conductuales de la alimentación producidos por el consumo de una dieta alta en grasa durante diez días.

\section{MÉTODO}

\section{Sujetos}

Se utilizaron 17 ratas macho adultas Wistar (provistas por la UNAM, FES Iztacala, $\mathrm{n}=17$ ) con un peso de 200-220 g al inicio de la investigación. Todos los procedimientos experimentales cumplieron con la Norma Oficial Mexicana (NOM-062-ZOO-1999) titulada "Especificaciones técnicas para la producción, cuidado y uso de animales de laboratorio".

\section{Situación experimental}

Los sujetos fueron colocados aleatoriamente en cajas-habitación individuales de acrílico transparente $(27 \times 37 \times 15 \mathrm{~cm})$, bajo un ciclo invertido de luz-oscuridad de $12 \times 12 \mathrm{~h}$ controlado automáticamente (inicio de la oscuridad a las 12:00 h) y temperatura del laboratorio a $22 \pm 1{ }^{\circ} \mathrm{C}$. El agua y el alimento estuvieron disponibles $a d$ libitum durante toda la investigación.

\section{Dietas}

Se utilizaron dos dietas con diferente contenido calórico, el grupo control tuvo acceso al alimento estándar en pellets ( $18 \%$ grasas, $24 \%$ proteínas y $58 \%$ carbohidratos; $3.1 \mathrm{kcal} / \mathrm{g}$, adquirido con Harlan Laboratories, México), mientras que al segundo grupo se le dio acceso a una dieta alta en grasa en pellets ( $45 \%$ grasas, $20 \%$ proteínas y $35 \%$ carbohidratos; $4.77 \mathrm{kcal} / \mathrm{g}$ Alimento para roedores D12451, adquirido con Research Diets Inc., New Brunswick, NJ).

\section{Diseño}

Se utilizó el diseño experimental de series cronológicas múltiples sin pretest. Este diseño plantea la posibilidad de efectuar varias observaciones a través del tiempo sobre una o más variables, bajo la consideración de que el efecto de la manipulación de la variable independiente tarda en expresarse. En este diseño se puede evaluar el efecto de la variable independiente a corto, mediano y largo plazo (Campbell \& Stanley, 1995); y, para este caso, la variable independiente fue el acceso durante diez días a la dieta alta en grasa, y las observaciones se realizaron en los días 1,5 y 10. 


\section{Aparatos}

Para grabar las pruebas conductuales se utilizó una cámara para baja intensidad de luz, una grabadora de video digital DGT-TBS1 y un monitor HICOM.

\section{Procedimiento}

Después del periodo de habituación al bioterio (3 días) se pesó diariamente a todos los animales y se determinó la porción de alimento ingerido cada 24 horas (se colectó el alimento derramado), durante diez días, al final del periodo de luz. Cada día se colocaba alimento fresco al inicio del periodo de oscuridad (12:00 h); y, además de obtener el consumo en gramos $(\mathrm{g})$, también se calculó la ingestión de calorías $(\mathrm{kcal} / \mathrm{g})$ y la tasa local de alimentación $(\mathrm{g} / \mathrm{min}$ $\mathrm{y} \mathrm{kcal} / \mathrm{min}$ ).

Se realizó una prueba conductual al inicio de la fase de oscuridad en los días 1, 5 y 10, pruebas que fueron videograbadas (60 min de duración) con una cámara para baja intensidad de luz, la misma que fue colocada en frente de las cajas-habitación. A partir de las videograbaciones se elaboraron los registros de duración continua para llevar a cabo el análisis de la SSC, esto conforme a lo sugerido por Halford et al. (1988). Específicamente, los 60 minutos de registro se dividieron en 12 periodos de 5 minutos y se calculó la duración (en segundos) de las siguientes categorías conductuales mutuamente excluyentes: alimentación - consumo de alimento-, descanso -inactividad con o sin ojos cerrados, con la cabeza de la rata en el piso de la caja-, acicalarse -lamer el cuerpo, pies y genitales, frotar la cara y los bigotes con las patas delanteras, o morder la cola- y actividad -conductas diferentes a comer o descansar, incluyendo locomoción, husmear y beber-.

En el décimo día, al término del registro conductual, se sacrificó a los animales con una mezcla de ketamina/ xilazina $(112.5 / 22.5 \mathrm{mg} / \mathrm{kg})$. Posteriormente, se diseccionó cuidadosamente el tejido adiposo gonadal, visceral y retroabdominal, y una vez extraído, se pesó de forma individual, siguiendo el método utilizado por Ravagnani et al. (2012).

\section{Análisis de datos}

Los datos del presente estudio se expresan en términos de la media \pm el error estándar de la media (SEM). Adicionalmente, se utilizaron pruebas estadísticas paramétricas dado que los datos cumplieron con los supuestos de normalidad y homocedasticidad. La ingestión de alimento y el peso corporal por día se analizaron con un ANOVA de dos vías (dieta $\mathrm{x}$ tiempo), mientras que los datos de la ingestión de alimento acumulada, el consumo de energía y el peso del tejido adiposo se compararon durante los diez días entre los grupos con la prueba $t$ de Student para muestras no relacionadas. También, se utilizó la duración (en segundos) de cada categoría de la SSC para calcular el área bajo la curva (AUC) y posteriormente se utilizó un ANOVA de dos vías (dieta $\mathrm{x}$ tiempo) para comparar cada una de las categorías. Por último, después de los ANOVA significativos se aplicó la prueba post hoc de Bonferroni para realizar las comparaciones múltiples. Para el análisis de los datos se utilizó el paquete estadístico GraphPad Prism Version 5.01 (GraphPad Software, San Diego, CA, USA), y el criterio para la significancia estadística fue de $p<.05$.

\section{RESULTADOS}

En esta sección se presentan los resultados de la evaluación del consumo de alimento (g por día y acumulado) y energía ( $\mathrm{kcal} / \mathrm{g}$ por día y acumulada), peso corporal (g acumulado) y tejido adiposo ( $\mathrm{g}$ al final del protocolo) durante los diez días que duró la exposición a las dietas control y alta en grasas. Posteriormente se muestran los resultados del análisis conductual detallado (tasa local de alimentación, SSC y áreas bajo la curva de las duraciones de la SSC), el cual se realizó específicamente al inicio de la fase oscura del ciclo de luz/oscuridad en los días 1, 5 y 10 de la exposición a las dietas. Todos los datos fueron expresados en términos de medias \pm el error estándar de la media (ESM).

Para conocer el curso temporal del consumo de alimento y de energía, estas dos variables se evaluaron durante diez días en dos grupos de ratas, uno con acceso a una dieta estándar de laboratorio y otro con una dieta alta en grasa. Como era de esperarse, el consumo de alimento difirió entre los grupos en función del tiempo (interacción dieta $\mathrm{x}$ tiempo), $F_{(9,150)}=4.07, p<.001$. La Figura 1 (panel izquierdo [arriba]) muestra que los sujetos de la dieta control consumieron cantidades crecientes de alimento, mientras que los sujetos experimentales mantuvieron relativamente estable el consumo de la dieta con alto contenido de grasas (factor tiempo), $F_{(1,150)}=199.5, p<.001$. Por otra parte, la ingestión acumulada de alimento (g) durante los diez días de observación fue significativamente menor en los sujetos de la dieta con alto contenido de grasas que en los sujetos del grupo control, $t_{(15)}=8.15, p<.0001$ (véase Figura 1, panel derecho [arriba]).

Paralelamente, se evaluó la ingesta energética en términos de calorías consumidas por gramo de alimento $(\mathrm{kcal} / \mathrm{g}), \mathrm{y}$ se encontró que los sujetos expuestos a la dieta con alto contenido de grasas tuvieron un consumo significativamente mayor en los primeros días de acceso al alimento (días 1-5, véase Figura 1, panel izquierdo [abajo]), aunque disminuyó posteriormente (interacción dieta x tiempo), $F_{(9,150)}=4.03$, 
$p<.01$. Este resultado indica que los sujetos tendieron a consumir menos alimento (días 6-10) para compensar el aumento de la ingesta calórica; sin embargo, la ingesta calórica acumulada $(\mathrm{kcal} / \mathrm{g})$ fue significativamente mayor en el grupo con dieta con alto contenido de grasas, $t_{(15)}=4.17$, $p<.001$ (véase Figura 1, panel derecho [abajo]).

Después de finalizar el periodo de exposición a las dietas, el peso corporal de los sujetos no mostró diferencias significativas entre los grupos (véase Figura 2 panel izquierdo [arriba]). Sin embargo, los sujetos con acceso a la dieta alta en grasas acumularon significativamente más tejido adiposo total, $t_{(15)}=8.15, p<.001$ (véase Figura 2, panel derecho [arriba]). Además, el tejido adiposo por regiones aumentó significativamente en los sujetos alimentados con la dieta alta en grasa (véase Figura 2, panel abajo) en las regiones gonadal, retroabdominal y visceral, $t_{(15)}=6.43, p<.0001, t_{(15)}=4.31$, $p<.0006$ y $t_{(15)}=7.43, p<.0001$, respectivamente.

Para caracterizar el patrón conductual de la alimentación producido por la dieta alta en grasa se analizó la SSC y la tasa local de alimentación en los días 1, 5 y 10 de exposición a las dietas. Los resultados muestran que el consumo de alimento por unidad de tiempo
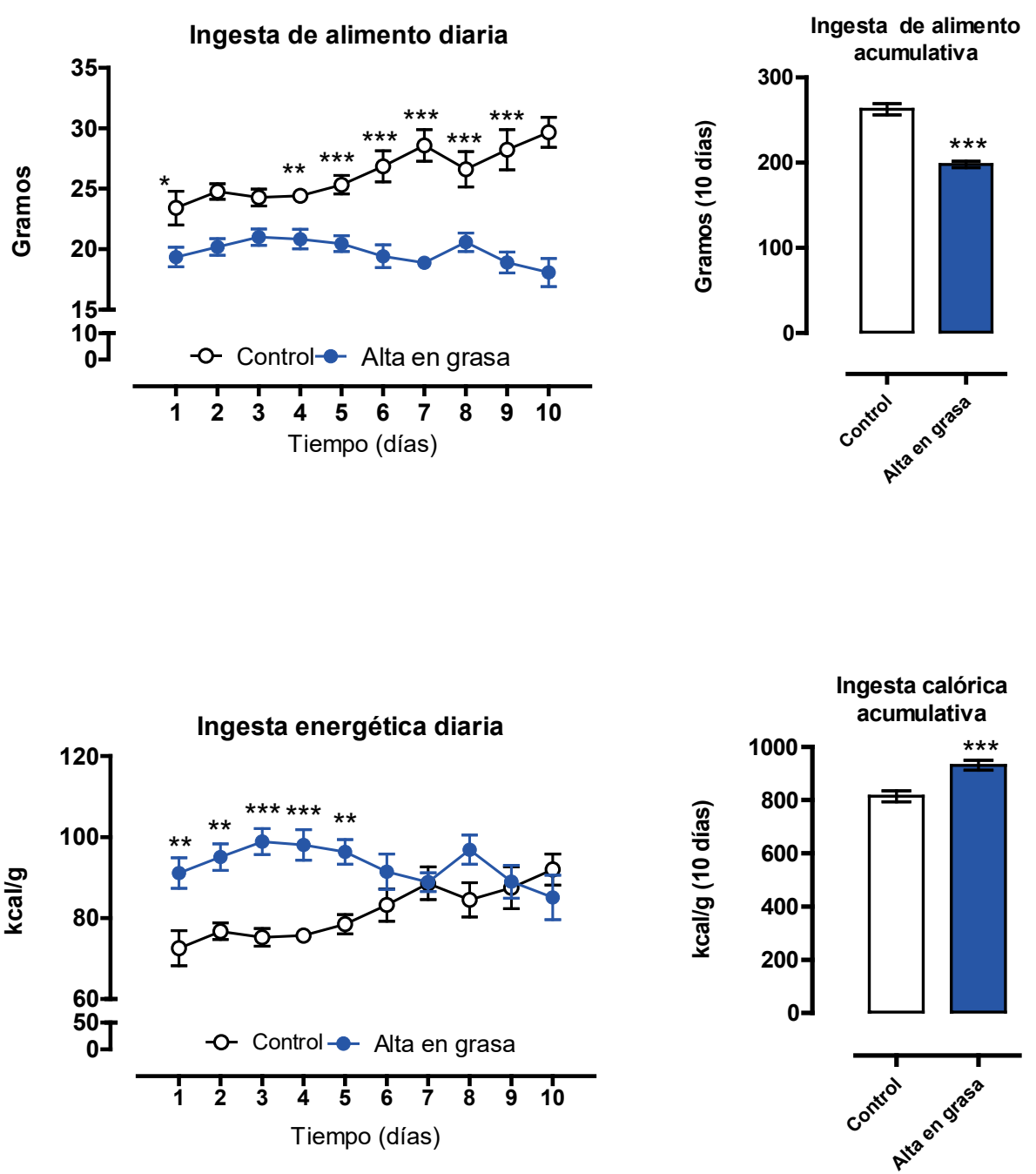

Figura 1. Dieta control vs. dieta alta en grasas. Curso temporal del consumo de alimento ( $\mathrm{g}$ ) y energía (kcal/g) durante diez días de exposición a las dietas control $(\mathrm{n}=9)$ y alta en grasa $(\mathrm{n}=8)$ (a la izquierda), consumo total de alimento y de energía (acumulado de los diez días de exposición) después del periodo de registro (a la derecha). Datos expresados en términos de medias \pm el SEM. $* p<.05, * * p<.01, * * * p<.001$. 


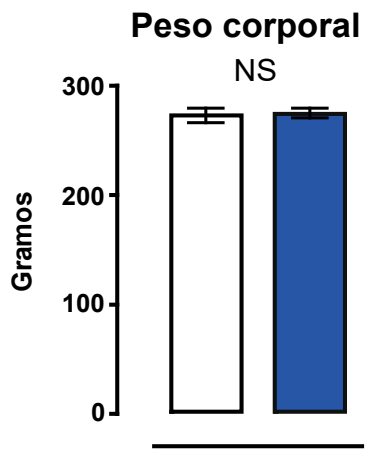

Tejido adiposo

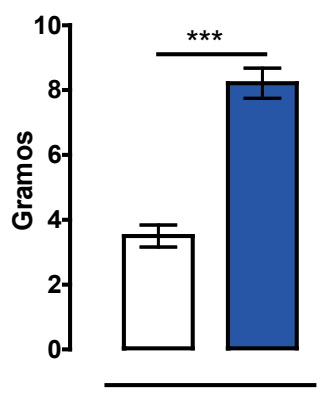

Tejido adiposo por regiones

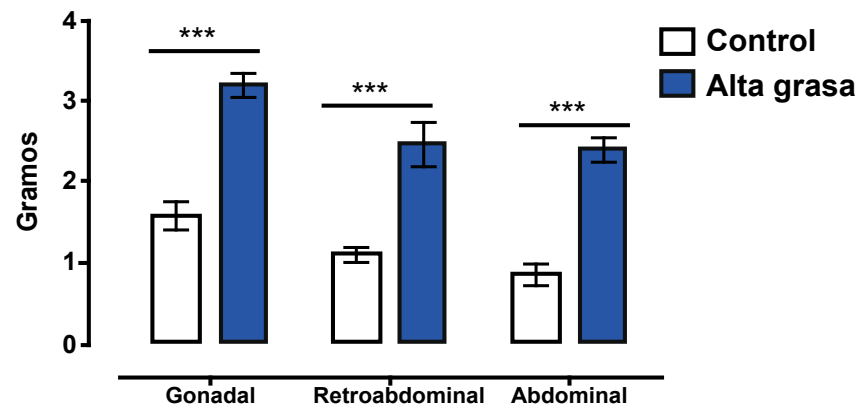

Figura 2. Dieta control vs. dieta alta en grasas. Peso corporal (panel izquierdo [arriba]) de los sujetos expuestos a las dietas control $(\mathrm{n}=9)$ y alta en grasa $(\mathrm{n}=8)$, acumulación de tejido adiposo total (panel derecho [arriba]) y acumulación del tejido adiposo por regiones (panel abajo), posterior a los diez días de registro. Datos expresados en términos de medias \pm el SEM. *** $p<.001 . \mathrm{NS}=$ no significativo.

( $\mathrm{g} / \mathrm{min}$ ) no difirió entre los grupos, pero el consumo de energía ( $\mathrm{kcal} / \mathrm{min})$ fue mayor en el grupo alimentado con la dieta alta en grasas (véase Tabla 1), este aumento alcanzó significancia estadística el día 10 de exposición a la dieta, $F_{(1,34)}=25.89, p<.001$.

El patrón temporal de la conducta alimentaria del grupo control en el primer día mostró una SSC típica, caracterizada por la presencia de episodios de alimentación intercalados con actividad durante los primeros periodos del registro; la transición entre la alimentación y el descanso ocurrió en el periodo 5, lo que indica que la saciedad postingesta se expresó a partir de los minutos 25-30 del registro conductual (véase Figura 3, panel izquierdo [arriba]).
Por su parte, el grupo con acceso a la dieta alta en grasas presentó en el primer día una SSC semejante a la de los sujetos control (véase Figura 3, panel derecho [arriba]), desarrollando una transición ordenada de la alimentación al descanso; sin embargo, la saciedad postingesta ocurrió después del séptimo periodo del registro (minutos 35-40). Además, los sujetos tuvieron mayor actividad y tendieron a descansar menos tiempo en el registro completo, lo que indica que la SSC progresó más lentamente que la de los sujetos control, demorando el proceso de satisfacción.

En el quinto día del protocolo alimentario, el grupo control desarrolló la secuencia de saciedad típica (véase Figura 3, panel izquierdo [medio]) semejante a la observada en el primer día, aunque con una progresión más rápida, 
y la transición de la alimentación al descanso se presentó en el periodo 4 del registro conductual (minuto 20). De la misma forma, los sujetos de la dieta alta en grasas mostraron una SSC típica, aunque más rápida que en el primer día de registro, y la transición de la alimentación al descanso se presentó en los periodos 3-4 del registro (minutos 15-20, véase Figura 3, panel derecho [medio]). Dado que ambos grupos de sujetos tendieron a disminuir el tiempo de actividad el quinto día de registro, es posible sugerir que los sujetos se alimentaron con mayor eficiencia, ya que desarrollaron el proceso de satisfacción en menos tiempo.

Finalmente, durante el registro en el décimo día, el grupo control presentó el patrón típico de la secuencia de saciedad conductual en los primeros 4 periodos (minutos 20-25), dando lugar al desarrollo natural del proceso de satisfacción (véase Figura 3, panel izquierdo [abajo]); mientras que el grupo alimentado con la dieta alta en grasas presentó, nuevamente, un patrón de saciedad semejante al del quinto día de registro (véase Figura 3, panel derecho [abajo]), con un desarrollo de la saciedad rápido, ya que la transición de la alimentación al descanso se estableció en el periodo 3 del registro (minutos 15-20).

Por otra parte, el análisis cuantitativo de la SSC se llevó a cabo mediante el cálculo del área bajo la curva de cada una de las categorías conductuales (alimentación, acicalarse, actividad y descanso), de esta forma, se evaluó la duración promedio de las categorías en cada grupo (véase Tabla 2). Así, se encontró que la duración de la alimentación fue afectada diferencialmente, dependiendo del número de días bajo la dieta (interacción dieta $\mathrm{x}$ tiempo), $F_{(2,36)}=3.28$, $p<.05$. En el grupo de dieta alta en grasas, los sujetos redujeron la duración de la alimentación con el paso de los días, y, de acuerdo con el procedimiento post hoc de Bonferroni, la reducción de la duración de la conducta de alimentación fue estadísticamente significativa el día 5 $(p<.05)$. Asimismo, la duración de la conducta de acicalamiento difirió significativamente (factor dieta) $F_{(1,35)}=4.41$, $p<.05$, pues la duración del acicalamiento aumentó en las ratas con la dieta alta en grasas. Finalmente, no se encontraron diferencias significativas entre los grupos en las categorías conductuales de actividad y descanso.

Tabla 1

Tasa local de alimentación ( $\mathrm{g} / \mathrm{min}, \mathrm{kcal} / \mathrm{min}$ ) en el inicio de la fase oscura (60 $\mathrm{min}$ ) en los días de registro 1, 5 y 10

\begin{tabular}{ccccccc}
\hline Categorías & \multicolumn{2}{c}{ Día 1} & \multicolumn{2}{c}{ Día 5} & \multicolumn{2}{c}{ Día 10} \\
Control & $\begin{array}{c}\text { Dieta alta } \\
\text { en grasa }\end{array}$ & Control & $\begin{array}{c}\text { Dieta alta } \\
\text { en grasa }\end{array}$ & $\begin{array}{c}\text { Control } \\
\text { grasa }\end{array}$ \\
\hline $\begin{array}{c}\text { Tasa local de alimentación } \\
(\mathrm{g} / \mathrm{min})\end{array}$ & $.30 \pm .04$ & $.40 \pm .07$ & $.42 \pm .05$ & $.42 \pm .03$ & $.46 \pm .06$ & $.66 \pm .12$ \\
$\begin{array}{c}\text { Tasa local de alimentación } \\
(\mathrm{kcal} / \mathrm{min})\end{array}$ & $.93 \pm .12$ & $1.89 \pm .33$ & $.93 \pm 12$ & $1.97 \pm .15$ & $1.46 \pm .19$ & $3.14 \pm .56^{* * *}$ \\
\hline
\end{tabular}

Nota. $* * * p<.001 v s$. mismo día grupo control.

Tabla 2

Área bajo la curva de la duración de las categorías de la SSC

\begin{tabular}{|c|c|c|c|c|c|c|}
\hline \multirow[b]{2}{*}{ Categorías } & \multicolumn{2}{|l|}{ Día 1} & \multicolumn{2}{|l|}{ Día 5} & \multicolumn{2}{|l|}{ Día 10} \\
\hline & Control & $\begin{array}{l}\text { Dieta alta en } \\
\text { grasa }\end{array}$ & Control & $\begin{array}{l}\text { Dieta alta en } \\
\text { grasa }\end{array}$ & Control & $\begin{array}{l}\text { Dieta alta en } \\
\text { grasa }\end{array}$ \\
\hline Alimentación (s) & $440.14 \pm 52.51$ & $529.42 \pm 75.91$ & $596.28 \pm 60.90$ & $345.833 \pm 69.21 *$ & $351.94 \pm 55.38$ & $360.62 \pm 81.41$ \\
\hline Descanso (s) & $1416.57 \pm 128.09$ & $756.83 \pm 102.37$ & $1266.71 \pm 303.83$ & $1616.00 \pm 277.64$ & $2036.19 \pm 187.95$ & $1499.71 \pm 323.36$ \\
\hline Acicalarse (s) & $571.00 \pm 31.81$ & $750.00 \pm 66.57$ & $741.50 \pm 158.56$ & $772.92 \pm 181.74$ & $507.14 \pm 81.37$ & $1023.87 \pm 199.49 *$ \\
\hline Actividad (s) & $872.50 \pm 88.46$ & $1264.42 \pm 125.28$ & $695.64 \pm 149.58$ & $559.58 \pm 96.51$ & $428.12 \pm 68.13$ & $572.813 \pm 115.68$ \\
\hline
\end{tabular}

Nota. ${ }^{*} p<.05$ vs. mismo día grupo control. 


\section{Dieta control}

Día 1

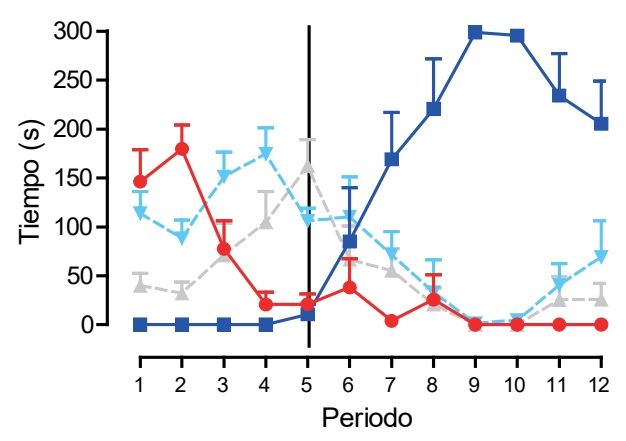

Día 5

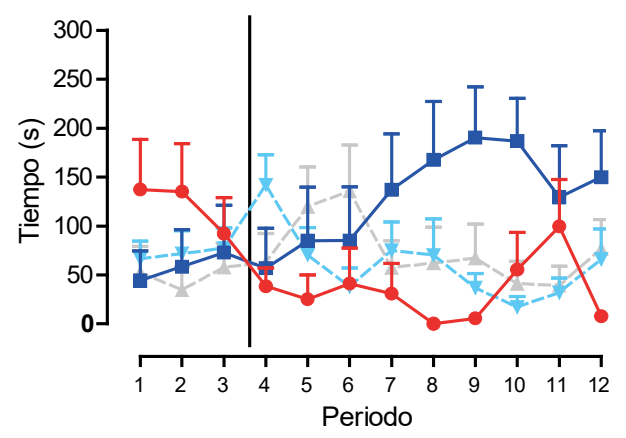

Día 10

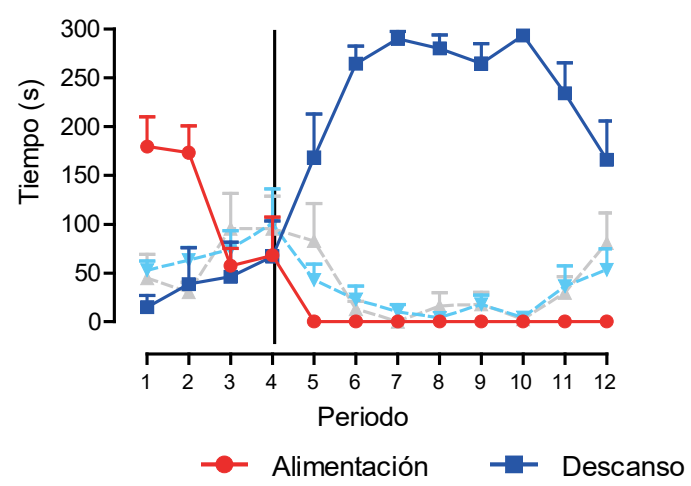

Dieta alta en grasa

Día 1

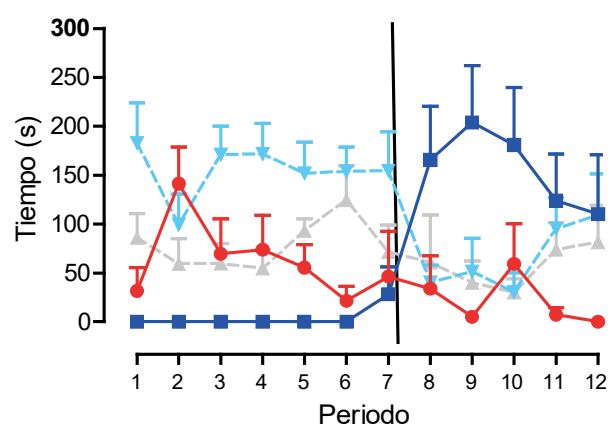

Día 5

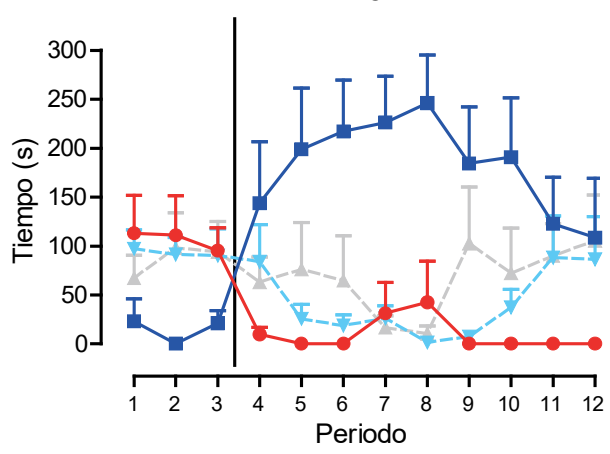

Día 10

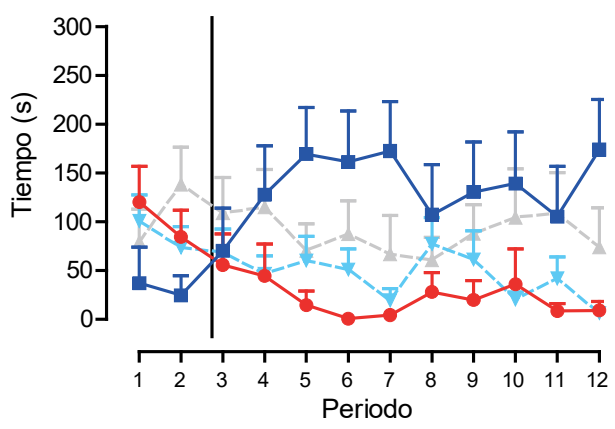

Figura 3. Curso temporal de la secuencia de saciedad conductual de los grupos expuestos a las dietas control $(\mathrm{n}=9) \mathrm{y}$ alta en grasa $(\mathrm{n}=8)$ en los días 1,5 y 10 del protocolo alimentario. El registro conductual de 60 min se dividió en 12 periodos de $5 \mathrm{~min}$. Los datos se expresan en términos de medias $\pm S E M$ de cada una de las categorías conductuales. La línea vertical indica la transición entre la alimentación y el descanso. 


\section{DISCUSIÓN}

El objetivo de esta investigación fue caracterizar los patrones conductuales y temporales de la alimentación de sujetos (ratas Wistar) expuestos a una dieta con alto contenido de grasas durante diez días. Así, se encontró que la exposición a la dieta alta en grasas solo aumentó la acumulación del tejido adiposo y la eficiencia de la alimentación (mayor consumo de energía por unidad de tiempo), sin incrementar el peso corporal de forma excesiva y sin alterar el patrón típico de la secuencia de saciedad conductual. Estos resultados sugieren que la exposición a las dietas con alto contenido de grasas puede producir cambios conductuales, específicamente en la eficiencia de la alimentación.

Esta investigación mostró que los sujetos expuestos a la dieta alta en grasa disminuyeron la ingesta de alimento (g) de forma voluntaria, lo que hace suponer que los sistemas regulatorios intervienen en la detección del aumento calórico ingerido y reaccionan para mantener la homeostasis. $\mathrm{Al}$ respecto, se sabe que el tamaño de los episodios alimentarios es, en primera instancia, controlado por procesos homeostáticos mediante el censo de las calorías consumidas (Cavanaugh, Schwartz \& Blouet, 2015). Por otra parte, el incremento del alimento ingerido al inicio de la investigación se acompañó del aumento de la ingestión de energía, lo que probablemente indujo la intensificación de las señales de saciedad y llevó a disminuir la cantidad (g) de alimento consumido para prevenir la ganancia de peso.

Igualmente, se sabe que en respuesta a la ingesta de alimento se estimula la liberación de las señales de saciedad, lo que puede hacerse a través de dos vías: la primera es la distensión del estómago al ingerir el alimento, que es meramente volumétrica (señales mecánicas); y la segunda, que es más compleja, implica un incremento de señales químicas, entre ellas la liberación de péptidos como la colecistoquinina (CCK) (Paulino, Darcel, Tome \& Raybould, 2008; Savastano \& Covasa, 2005), el péptido glucagón-like 1 (GLP-1) (Williams, Baskin \& Schwartz, 2009) y el péptido YY (PYY) (Batterham et al., 2002). Estas señales van del estómago al cerebro, y, específicamente, el hipotálamo recibe e integra esta información para promover la satisfacción y la saciedad, por lo que estas señales fisiológicas participan en la regulación del tamaño de los episodios alimentarios (cantidad de alimento ingerido).

Sin embargo, a pesar de disminuir la ingestión de alimento, los sujetos alimentados con la dieta alta en grasa consumieron más calorías, y esto contribuyó con el aumento de la acumulación de tejido adiposo gonadal, retroabdominal $\mathrm{y}$ visceral, sin que aumentara significativamente el peso corporal. Investigaciones previas señalan que el aumento del tejido adiposo es una condición que precede al aumento de peso y a la presentación de síntomas relacionados con la obesidad (Bjursell et al., 2008); y aunque la obesidad es una condición multicausal en la que se incluyen factores ambientales, genéticos y conductuales, su génesis se ha asociado principalmente al consumo de alimentos con alta densidad energética y la disminución del gasto energético (Ludmilla et al., 2017). De tal forma, el aumento de la ingesta calórica y la acumulación de grasa corporal podrían ser la etapa previa a la ganancia de peso y el desarrollo propiamente de la obesidad.

Por otro lado, contrario a la idea de que las dietas con alto contenido de grasas promueven que los episodios alimentarios sean menos frecuentes, pero de mayor duración (Melhorn et al, 2010), en el presente estudio se encontró que los sujetos experimentales no aumentaron el tiempo de alimentación, aunque sí aumentaron la ingesta calórica en la segunda mitad de la exposición a la dieta, ya que a partir del día 6 y hasta el día 10, las ratas con acceso a la dieta alta en grasas disminuyeron la ingestión de alimento en términos de gramos y duración. Sin embargo, a pesar de que el consumo total de alimento durante los diez días de exposición a la dieta experimental fue menor que el de los controles, la ingesta calórica acumulada fue significativamente mayor.

Así como la SSC ha sido utilizada para caracterizar el efecto de las manipulaciones farmacológicas y no farmacológicas sobre la conducta alimentaria de roedores, la preservación del patrón (alimentarse, actividad, acicalarse y descanso) se ha asociado al proceso de satisfacción producto de la postingesta de alimento. De tal forma, en el presente estudio se realizó la caracterización de los patrones conductuales asociados a la sobreingestión calórica de los sujetos expuestos a la dieta con alto contenido de grasas, sin embargo, se encontró que la SSC no se alteró en términos de su morfología (secuencia de las categorías conductuales) (Halford et al., 1998; Tallett et al., 2009), pero sí en su eficiencia.

Lo anterior implica que los sujetos mostraron una capacidad limitada para disminuir la cantidad ingerida de alimento calóricamente denso, ya que aunque la duración de la alimentación en el día 5 de exposición a la dieta fue menor que la de los controles, al final del protocolo los sujetos consumieron cantidades mayores de energía por unidad de tiempo. Así, uno de los hallazgos más importantes del presente estudio lo constituye la observación del aumento de la eficiencia alimentaria al final de la exposición a la dieta experimental.

Finalmente, los sujetos que consumieron la dieta alta en grasas ingirieron cantidades mayores de energía por unidad 
de tiempo y la SSC, aunque no se alteró en su patrón básico (progresión ordenada de la alimentación al descanso), fue cada vez más rápida, y aunque el proceso de satisfacción se desarrollaba en menos tiempo, los sujetos consumían mayores cantidades de energía en la prueba conductual al inicio de la fase de oscuridad (registro de 60 minutos).

En síntesis, la exposición a la dieta alta en grasa favoreció el aumento del tejido adiposo, sin producir aumento del peso corporal. Aunque se observó la reducción de la ingestión de alimento en los sujetos que fueron expuestos a la dieta alta en grasa, esto no fue suficiente para prevenir el aumento del consumo calórico, que resultó significativamente mayor en los sujetos expuestos a la dieta con alto contenido de grasas.

Los resultados de esta investigación apuntan a que el consumo de dietas altas en grasas puede contribuir en el cambio de los patrones conductuales, particularmente en aquellos que participan en la regulación de la eficiencia de la alimentación (consumo energético por unidad de tiempo). Futuras investigaciones podrían estudiar la relación entre los cambios en las señales saciatorias en regiones cerebrales que se producen por la exposición a dietas con alto contenido de grasas y los mecanismos de control del apetito (eficiencia de la alimentación), para tener un mejor entendimiento de los mecanismos neuroquímicos que favorecen el desarrollo del sobrepeso y la obesidad.

\section{REFERENCIAS}

Batterham, R. L., Cowley, M. A., Small, C. J., Herzogk, H., Cohen, M. A., ... Bloom, S. R. (2002). Gut hormone PYY336 physiologically inhibits food intake. Nature, 418(6898), 650-654. DOI:10.1038/nature00887.

Bjursell, M., Gerdin, A-K, Lelliott, C. J. Egecioglu, E., Elmgren, ... Bohlooly-Y, M. (2008). Acutely reduced locomotor activity is a major contributor to Western diet-induced obesity in mice. American Journal of Physiology Endocrinology and Metabolism, 294(2), E251-E260. DOI:10.1152/ ajpendo.00401.2007.

Campbell, D. T., \& Stanley, J.C. (1995). Diseños experimentales y cuasiexperimentales en la investigación social (7a ed.). Buenos Aires, Argentina: Amorrortu editores.

Carlin, J., McKee, S., Hill-Smith, T., Grissom, N. M., George, R., Lucki, I., \& Reyes, T. M. (2016). Removal of high-fat diet after chronic exposure drives binge behavior and dopaminergic dysregulation in female mice. Neuroscience, 326 , 170-179. DOI:10.1016/j.neuroscience.2016.04.002.

Cavanaugh, R. A., Schwartz, G. J., \& Blouet, C. (2015). Highfat feeding impairs nutrient sensing and gut brain integration in the caudomedial nucleus of the solitary tract in mice. Plos One, 10(3), 1-12. DOI:10.1371/journal.pone.0118888.
Chandler, P. C., Viana, J. B., Oswald, K. D., Wauford, P. K., \& Boggiano, M. M. (2005). Feeding response to melanocortin agonist predicts preference for and obesity from a high-fat diet. Physiology \& Behavior, 85(2), 221-230. DOI:10.1016/j.physbeh.2005.04.011.

Fordahl, S. C., Locke, J. L., \& Jones, S. R. (2016). High fat diet augments amphetamine sensitization in mice: Role of feeding pattern, obesity, and dopamine terminal changes. Neuropharmacology, 109, 170-182. DOI:10.1016/j.neuropharm.2016.06.006.

Halford, J., Wanninayake, C., \& Blundell, J. (1998). Behavioral satiety sequence (BSS) for the diagnosis of drug addiction on food intake. Behavioral, Biochemistry \& Behavior, 61(2), 159-168. DOI:10.1016/S0091-3057(98)00032-X.

Hennink, S. D., \& Maljaars, P. W. J. (2013). Fats and satiety. En J. E. Blundell \& F. Bellisle (Eds.), Satiation, satiety and the control of food intake. Theory and practice (pp. 143-165). United Kingdom: Woodhead Publishing Limited. DOI:10.1 533/9780857098719.3.143.

Honma, K., Hikosaka, M., Mochizuki, K., \& Goda, T. (2016). Loss of circadian rhythm of circulating insulin concentration induced by high-fat diet intake is associated with disrupted rhythmic expression of circadian clock genes in the liver. Metabolism clinical and Experimental, 65(4), 482491. DOI:10.1016/j.metabol.2015.12.003.

Kentish, S., Li, H., Philp, L. K., O’Donnell, T. A., Isaacs, N. J., ... Amanda J. (2012). Diet-induced adaptation of vagal afferent function. Journal of Physiology, 590(1), 209-221. DOI: 10.1113/jphysiol.2011.222158.

La Fleur, S., Van Rozen, A., Luijendijk, M., Groeneweg, F., \& Adan, R. (2010). A free-choice high-fat high-sugar diet induces changes in arcuate neuropeptide expression that support hyperphagia. International Journal of Obesity, 34(3), 537-546. DOI:10.1038/ijo.2009.257.

Leibowitz, S., \& Alexander, T. J. (1998). Hypothalamic serotonin in control of eating behavior, meal size, and body weight. Biological Psychiatry, 44(9), 851-864. DOI:10.1038/ ijo.2009.257.

López-Alonso, V. E., Mancilla-Díaz, J. M., Rito-Domingo, M., González-Hernández, B., \& Escartín-Pérez, R. E. (2007). The effects of 5- $\mathrm{HT}_{1 \mathrm{~A}}$ and $5-\mathrm{HT}_{2 \mathrm{C}}$ receptor agonists on behavioral satiety sequence in rats. Neuroscience Letters, 416(3), 285-288. DOI:10.1016/j.neulet.2007.02.026.

Ludmilla M. T., Dias B. I., Pereira, de C. F., Barthichoto, M., Le Sueur-Maluf, L., \& Machado, de O.C.A. (2017). The effects of calorie-matched high-fat diet consumption on spontaneous physical activity and development of obesity. Life Sciences, 179, 30-36. DOI:10.1016/j.lfs.2017.04.017.

Maljaars, J. (2013). Overeating makes the gut grow fonder; new insights in gastrointestinal satiety signaling in obesity. Current Opinion Gastroenterology, 29(2), 177-183. DOI:10.1097/MOG.0b013e32835d9fe0.

Mancilla-Díaz, J. M, Escartín-Pérez, R. E., López-Alonso, V. E., Floran-Garduño, B., \& Romano-Camacho, B. (2005). 
Role of 5- $\mathrm{HT}_{1 \mathrm{~A}}$ and 5-HT $\mathrm{H}_{1 \mathrm{~B}}$ receptors in the hypophagic effect of 5-HT on the structure of feeding behavior. Medical Science Monitor, 11(3), BR74-BR79.

Melhorn, S. J., Krause, E. G., Scott, K. A., Mooney, M. R., Johnson, J. D., \& Woods, S. C. (2010). Acute exposure to a high-fat diet alters meal patterns and body composition. Physiology \& Behavior, 99(1), 33-39. DOI:10.1016/j.physbeh.2009.10.004.

Mul, J. D., Spruijt, B. M., Brakkee, J. H., \& Adan, R. A. (2013). Melanocortin MC4 receptor-mediated feeding and grooming in rodents. European Journal of Pharmacology, 719(1-3), 192-201. DOI:10.1016/j.ejphar.2013.04.060.

Paulino, G., Darcel, N., Tome; D., \& Raybould, H. (2008). Adaptation of lipid-induced satiation is not dependent on caloric density in rats. Physiology \& Behavior, 93(4-5), 930-936. DOI:10.1016/j.physbeh.2007.12.015.

Ravagnani, F. C., Ravagnani, C. F., Braga, N. J. A., Azevedo V. F., Zavala, Z. A. A., Habitante, C. A., \& Massaschi, I. C. (2012). Effects of high fat diets with baru extract and chocolate on adipocyte area of rats subjected to physical exercise. Revista Brasileira de Medicina do Esporte, 18(3), 190-194. DOI:10.1590/S1517-86922012000300011.

Sáinz, N., Barrenetxe, J., Moreno-Aliaga, M. J., \& Martínez, J. A. (2015). Leptin resistance and diet-induced obesity: central and peripheral actions of leptin. Metabolism, 64(1), 3546. DOI:10.1016/j.metabol.2014.10.015.

Savastano, D. M., \& Covasa, M. (2005). Adaptation to a highfat diet leads to hyperphagia and diminished sensitivity to cholecystokinin in rats. Journal of Nutrition, 135(8), 19531959.

Sherman, H., Genzer, Y., Cohen, R., Chapnik, N., Madar, Z., \& Froy O. (2012). Timed high-fat diet resets circadian metabolism and prevents obesity. Faseb Journal, 26(8), 34933502. DOI:10.1096/fj.12-208868.
Tallett, A., Blundell, J., \& Rodgers, R. (2009). Night and day: diurnal differences in the behavioural satiety sequence in male rats. Physiology \& Behavior, 97(1), 125-130. DOI:10.1016/j.physbeh.2009.01.022.

Tejas-Juárez, J. G., Cruz-Martínez, A. M., López-Alonso, V. E., García-Iglesias, ... Escartín-Pérez, R. E. (2014). Stimulation of dopamine D4 receptors in the paraventricular nucleus of the hypothalamus of male rats induces hyperphagia: Involvement of glutamate. Physiology \& Behavior, 133, 272-281. DOI: 10.1016/j.physbeh.2014.04.040.

Terry, P., Gilbert, D. B., \& Cooper, S. J. (1995). Dopamine receptor subtype agonists and feeding behavior. Obesity Research, 3(4), 515S-23S. DOI:0.1002/j.1550-8528.1995. tb00221.x.

Williams, D., Baskin, D. G., \& Schwartz, M. W. (2009). Evidence that intestinal glucagon-like peptide-1 plays a physiological role in satiety. Endocrinology, 150(4), 16801687. DOI:10.1210/en.2008-1045.

Woods, S., Seeley, R. J., Rushing, P. A., D'Alessio, D., \& Tso, P. (2003). A controlled high-fat diet induces an obese syndrome in rats. Journal of Nutrition. 133(4), 1081-1087.

Yu, Y., Wu, Y., Patch, C., Wu, Z., Szabo, A., Li, D., \& Huang, X-F. (2013). DHA prevents altered 5-HT1A, 5-HT2A, $\mathrm{CB} 1$ and GABAA receptor binding densities in the brain of male rats fed a high-saturated-fat diet. Journal of $\mathrm{Nu}$ tritional Biochemistry, 24(7), 1349-1358. DOI:10.1016/j. jnutbio.2012.11.002. 